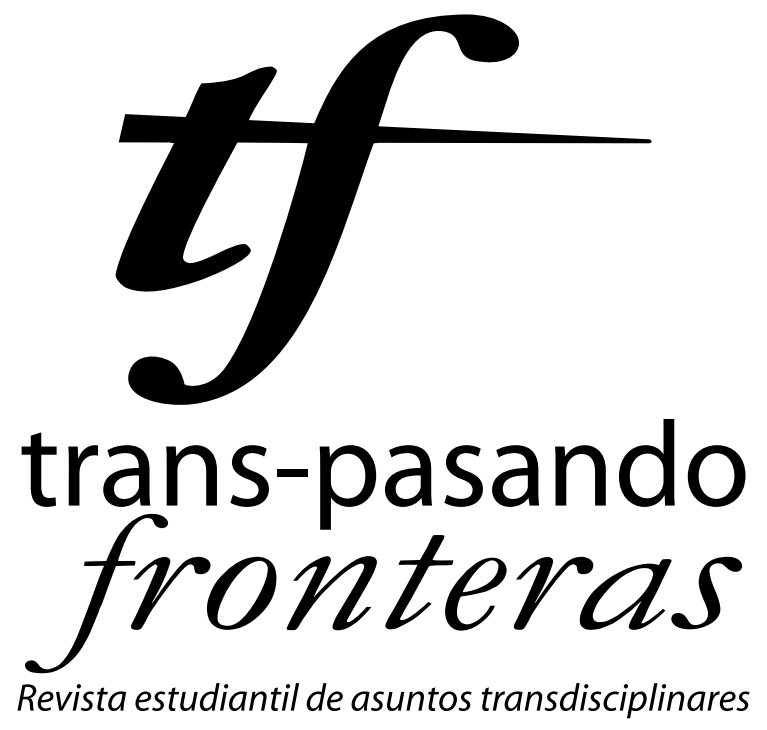

Una publicación de

Estudios

Interdisciplinarios

Jurídicos, Sociales

000 y Humanistas

ICESS

FACULTAD DE

DERECHO Y CIENCIAS

SOCIALES

然 UNIVERSIDAD 


\title{
Oriente y occidente. Chris Patten a la luz del estructuralismo del sistema político internacional de Kenneth Waltz
}

\author{
Juan Felipe Chavarriaga \\ (jf.chavarriaga@gmail.com)
}

PATTEN, Chirs (1999). East and West. Pan Macmillan, New Ed edition, 384 pp.

Este libro es difícil de definir en pocas palabras. La actitud franca y a veces controversial con la que Patten relata su experiencia y opiniones respecto de la forma de negociar con un país como China, opinando directamente de las políticas y los tomadores de decisión de esta república directamente, resulta sorprendente y en algunos extremos un tanto divertido, teniendo en cuenta la forma mediante la cual el autor se refiere a diferentes experiencias, historias y personas relacionadas con el proceso que describe.

El libro claramente se centra en un suceso que el mismo autor llama "histórico": los últimos años de Hong Kong bajo la soberanía de Gran Bretaña, y el proceso de transición que se llevó a cabo para entregar dicha soberanía a la República Popular de China.

Desarrollar un análisis desde el punto de vista del estructuralismo se torna un tanto arduo si tenemos en cuenta que Patten describe su labor, las tensiones, los encuentros, y las circunstancias que tuvo que afrontar, bajo posiciones y factores que normalmente Kenneth Waltz desecharía en el camino hacia el estudio objetivo de las relaciones entre los intereses de China, Gran Bretaña, e incluso en algunos momentos los intereses de Hong Kong propiamente, en una cuestión que era llamada como "el asunto de las tres piernas (The Three legged question)". Aún así, encuentro muy interesante poner en discusión las dos formas 
de análisis (la que realiza Patten y la que propone Waltz) para determinar a partir de los dos puntos de vista, cuál brinda conclusiones o un diagnóstico de dicha relación con un carácter satisfactorio.

A manera de información previa, en primer lugar se debe recordar que la Teoría Estructuralista de las Relaciones Internacionales, también conocida como Neorrealismo, planteada por Kenneth Waltz, entiende a los diferentes Estados como unidades independientes que interactúan entre sí, según la estructura bajo la que se encuentren ordenados. Esta estructura es formada a partir de un criterio de organización o principio ordenador, el cual es formado a su vez, por la coexistencia que se dé entre las unidades según las diferentes capacidades o funciones dentro del sistema internacional que cada una posea. En este sentido, un cambio en la distribución de capacidades o en el principio ordenador son factores que provocan cambios en la estructura y por ende, cambios en el funcionamiento del sistema internacional. Es necesario mencionar que en esta teoría, el poder es medido según el número de capacidades que posea cada unidad, ya que la unidad que más capacidades tenga, tendrá el poder de influir en la formación de la estructura del sistema internacional. Finalmente cabe destacar, que teniendo en cuenta que cada tipo de estructura determina las interacciones entre los Estados o unidades, el estudio y análisis de estas puede verse como una útil herramienta a la hora de intentar predecir o categorizar los comportamientos que puedan adoptar diferentes Estados en determinadas situaciones.

En segundo lugar, considero importante tener en cuenta la temporalidad en la que se desenvuelve el relato descrito por Patten; la década de los 90 está perfilada hacia las políticas comunistas y claramente antidemocráticas del lado chino, mientras que los intereses británicos se encaminan como en gran parte de la historia reciente, hacia la defensa de las libertades individuales, la democracia y el libre mercado como factores y principios inobjetables si la intención es propiciar el desarrollo de la sociedad; esto es de suprema importancia para poder analizar las tensiones entre estos 2 poderes.

Patten durante el texto menciona inicialmente aspectos básicos de la sociedad relacionados con la formulación de políticas, como una descripción de la situación de la colonia que él recibía para gobernar y posteriormente aclara las materias en las cuales su administración mostró resultados 
positivos. El reto para el gobierno británico era poder tener la certeza de garantizar a los ciudadanos de Hong Kong, que después del traspaso hacia la soberanía china, seguirían disfrutando de las libertades que poseían en manos británicas, y aun mas allá, que serian regidos por un "régimen citadino" democrático. El primer paso ya se había tomado a finales de los años 80 , cuando en una declaración conjunta, los dos gobiernos habían acordado las bases sobre las cuales se haría el traspaso de Hong Kong hacia su "patria madre". El autor relata las tensiones que se vivieron con los diplomáticos chinos desde una perspectiva que para Waltz sería subjetiva, pero que considero no pierde importancia si tenemos en cuenta el simbolismo de los actos y declaraciones por parte de los asiáticos. Entre estas dos naciones, es imprescindible para el análisis estructuralista, tener en cuenta la ambigüedad del termino relación y adoptar la definición que la concreta como la posición de una unidad frente a la otra; entendiendo tanto a China como a Gran Bretaña como unidades diferenciadas. Esto claramente sugiere la necesidad de evadir los atributos de dichas unidades tales como la clase de líderes políticos y/o compromisos ideológicos, y más retador aún, evadir o abstraer las rela- ciones como interacciones culturales, económicas y políticas por ejemplo.

En este sentido nos encontramos ante un problema, teniendo en cuenta que el texto en gran parte gira alrededor de justamente los factores que se proponen evitar en la mirada estructuralista. Si efectivamente dejáramos de lado lo anteriormente mencionado, entonces simplemente nos encontraremos ante los "principios de disposición" de las partes; que en este caso específico corresponden a la intención de cada una de ellas acerca del futuro de Hong Kong. Siguiendo con la mirada de Waltz, deberíamos caracterizar a las unidades que se estudian en términos de la especificación de funciones de las partes diferenciadas; que en este caso corresponde a las condiciones bajo las cuales Gran Bretaña estaba dispuesta a seguir con los planes de la declaración conjunta y entregar la soberanía, teniendo o exigiendo como limite el mes de junio de 1997 para finalizar la transición y China estaba dispuesta a recibir a Hong Kong y cumplir con acuerdos o deudas impuestas por el gobierno británico. Esto lo podemos ver expresamente en el texto por ejemplo, cuando Patten habla de la necesidad de construir un nuevo aeropuerto en Hong Kong, y la posterior preocupación de los 
británicos relacionada con la actitud que tomaría el gobierno chino frente a la obligación de pagar una deuda adquirida para llevar a cabo dicho proyecto. Entonces es en este punto que se debe tener en cuenta la capacidad de cada nación de llevar a cabo su propósito. Pero temo que para este objetivo, necesariamente se debe analizar el simbolismo de los actos chinos que mencionaba anteriormente, lo cual desvirtúa en parte el argumento que defiende la inutilidad de dichos factores en el análisis sistémico de Waltz. Es decir, no se puede simplemente pasar por alto la actitud del gobierno chino frente al representante británico (Patten) el día en que por ejemplo éste visito Pekín con la intención de lograr un acercamiento con la cerrada posición de los asiáticos con relación al futuro de Hong Kong; la descortesía de no recibirlo en el aeropuerto, el perfil constante de China a sabotear las conversaciones a través del libre acceso a periodistas y como si fuera poco, la situación "mecánica” que sufrió el auto de Patten de vuelta a su hotel son "casualidades" o "eventos" que tienen un claro significado, y un claro objetivo. Como tampoco se pueden pasar por alto los argumentos de los británicos al asegurar (con razón) que el desarrollo que había logrado su anteriormente colonia pudo haber sido posible únicamente por la vigencia del sistema capitalista en la ciudad, sumado a la defensa de las libertades individuales y otros factores.

Otro aspecto que el análisis estructuralista no permite, pero que considero de gran importancia, es el económico. Waltz dice que este aspecto se debe obviar. Pero, ¿Cómo obviar un factor que conforma inevitablemente los argumentos y las capacidades de cada una de las partes? Claramente los británicos pensaban en este factor (el económico) como un punto que debía mostrarse como un logro del ambiente de inversión sano y libre que vivía Hong Kong; y por su lado, incluso los chinos utilizaron la punta de lanza económica para arremeter en contra de las intenciones del gobierno británico cuando trataban de convencer a los empresarios que tenían intereses y relaciones comerciales entre Londres y Hong Kong para que presionaran a sus gobernantes (al punto de amenazar con retirar la financiación al partido de Patten) si no se retractaban de las intenciones del, en ese entonces gobernador de la colonia. Este por lo tanto es otro punto que a mi modo de ver, desvirtúa la viabilidad de la propuesta de Waltz, o al menos lo hace para este caso específico.

En el tercer y último punto del análi- 
sis sistémico que plantea el estructuralismo, nos enfrentamos a la distribución de las capacidades de cada parte, lo que supuestamente brinda una explicación de la situación en términos posicionales y no en términos de atributos. Pero como anteriormente se dijo, esto no es posible ya que las capacidades, que son las que definen la posición de una unidad frente a la otra, en este caso están directamente relacionadas con los atributos de un lado y de otro.

Este documento únicamente tuvo en cuenta a dos partes como unidades sujetos del análisis (China y Gran Bretaña), pero es claro que, en caso de tener en cuenta la cuestión de las tres piernas (llamada así por los Chinos) que se menciona más arriba, y adicionar a este "mini sistema" la participación activa de los representantes de los intereses propios de Hong Kong en las conversaciones, veríamos como se complejiza aún más este escenario, y todo, una vez mas, en función de atributos que Waltz propone obviar.

Han pasado un poco más de 15 años desde que Hong Kong entró a ser parte de la soberanía de los chinos. Y todavía podemos ver que el análisis hecho en su momento, y expresado abiertamente en el libro por Chris Patten visiblemente lo llevó a tener un diagnóstico adecuado de la situación, y por ende a tomar las decisiones correctas; aspecto que podemos ver en el actual protagonismo de la antigua colonia en el mercado internacional y en las dinámicas sociales de las personas, empresarios y trabajadores que habitan Hong Kong. 